\title{
V.
}

\section{Nietzsche und die Entstehung der sittlichen Vorstellungen.}

\author{
Von
}

Prof. Dr. Paul Schwartzkopff in Wernigerode.

\section{Das individuelle Leben in seiner Stellung zum Gesamt- leben unter dem sittlichen Gesichtspunkt.}

1. Nietzsches sittlicher Individualismus.

Schopenhauer zuerst hat der buddhistischen Ethik in weiteren Kreisen der modernen Gesellschaft, vor allem unter höher Gebildeten, eine gewisse Vorherrschaft erkämpft. Die Zeichen mehren sich, daß sich diese allmählich ihrem Ende nähert. Die in ihr enthaltene Einseitigkeit tritt immer deutlicher hervor. In dieser Hinsicht gebührt dem Einsiedler von Sils Maria ein Verdienst.

Der Buddhismus will die Vernichtung des Selbst. Für ein Selbst ist aber Selbstvernichtung sinnlos. Höchstens dürfte vielleicht von Selbstverleugnung, Selbstbeherrschung, Selbsterziehung, Selbstzucht die Rede sein. Aber es soll im Grunde überhaupt kein Selbst geben. Der Unterschied zwischen Ich und Du ist angeblich nur scheinbär. In Wahrheit sollen beide dasselbe sein.

Nun, wenn ich wirklich der Andre bin, wird danach freilich auch der Altruismus zum Egoismus. Aber es würde dann in der Tat der Wahrheit entsprechen, wie der Buddhismus es fordert, den Anderen als mein Selbst zu lieben.

Nur mögen "Ich" und „Du" in dem gleichen Grunde wurzeln. Aber so ohne weiteres der Andere bin ich eben nicht. Es gibt kein "alter ego“, wenn man es genau nimmt. Das glaubt auch 
kein unbefangener Mensch. Daher kann ein gesunder Altruismus nur darin bestehen, daß man den Anderen wie sein Selbst liebt. Nämlich als einen, der mir gleichgeartet ist und demselben Urquell entstammt. So hat der, in welchem sich die Nächstenliebe selber verkörperte, uns mit Wahrheit angewiesen, den Nächsten zu lieben wie uns selbst.

Freilich: der Buddhismus verzichtet ja auf eigentliche Nächstenliebe. Ich soll gegen andere Menschen keinen $\mathrm{HaB}$, jedoch auch keine Liebe, sondern Mitleid hegen. Aber selbst diese Anweisung geht schon über das hinaus, was der Buddhismus fordern und leisten kann. Im strengen Sinne kann er den Altruismus selbst in dieser abgeschwächten Form nicht begründen. Hier fordert in Buddha und seinen Anhängern der gute Mensch sein Recht, dem Buddhismus zum Trotz.

Denn das Individuum ist ja nach Gautama nichtig, bloßer Schein. Was hat nun das Rechtverhalten mit bloßem Scheine tun? Ebensowenig bei anderen, wie bei mir selbst. Die ganze Welt ist für den Buddhisten wertlos, so auch die Individuen Weshalb dann die wertlosen Nächsten überhaupt der Beachtung würdigen? Endlich soll auch das Leid zur Maja gehören, zu der großen Welttäuschung. Was für einen Sinn hat es nun, mitzuleiden und sich dadurch noch ausdrücklich mit dem Scheine zu verbinden! Dies widerspricht vielmehr dem Ziele des Buddhismús selbst, nämlich: den Schein zu vernichten.

Aber in diesem Widerspruch tritt der richtige Instinkt ans Licht. Der Instinkt, daß sich in den Individuen als solchen, die doch allein alles Leid und alle Freude angeht, im Grunde ein wertvolles Wesen äußert, welches es auch in den Erfahrungen des individuellen Lebens nicht bloß mit Schein, sondern mit Wahrheit zu tun hat.

Soll irgendwelche Teilnahme mit Menschen, wenn es auch nur Mitleid ist, Sinn haben, dann ist die Voraussetiung, daß die individuellen Menschenseelen als solche nicht wertlos, geschwoige denn bloßer Schein sind. Wenn der Einzelne durchaus nichtig ist, sind dies eben alle Einzelnen. Dann ist auch ihre Summe, die Menschheit, nichtig. 
Diesen Wert des Individuums hat in neuester Zeit niemand so energisch belont, wie Nietzsche. So bildet seine kraftvolle Verwertung des Individualismus für die Ethik die Reaktion auf die Einsoitigkeit des buddhistischen Altruismus, der das Individuum aufhebt. Dies sollte man nicht mehr verkennen. Man sollte liebor die auf diesem Gebiete von Nietzsche entdeckten Wahrheiten für den Ausbau der Sittenlehre fruchtbar machen. Vor allem für die so schwierige und noch kaum begonnene Untersuchung über die Entstehung der sittlichen Vorstellungen.

Freilich: Einseitig ist Nietzsches Auffassung dieses Vorgangs durch und durch. Beginnen wir gleich mit der Hauptsache. Es ist allerdings das Recht unseres Philosophen, die Selbstliebe wieder in ihr gebührendes Licht zu stellen. Ohne echte Selbstschätzung und Selbstliebe ist keine echte Schätzung und Liebe des. Nächsten möglich. Denn den Wert, dessen das Individuum als solches in mir ermangelt, kann es auch in anderen nicht besitzen. Darum leuchtet in einem Brennpunkte des Christentums der Selbstwert der Menschenseele.

Dies zugestanden, so heißt es aber doch das Kind mit dem Bade ausschütten, wenn Nietzsche nun allen Altruismus grundsätzlich durch den Egoismus auflöst und diesen als allein berechtigt hinstellt.

Durch seinen Kultus des Genius und am Ende des Übermenschen setzt er dies Mißverhältnis zwar wieder etwas ins Gleichgewicht. Aber nur auf Kosten seiner eigenen Lehre." Die Gesellschaft soll garnichts gelten; der Genius alles. Ein Volk soll nur dazu da sein, um ein paar Genien, die Menschleit, um endlich den Übermenschen hervorzubringen.

Nun ist ja nicht zu leugnen, daß. der wertvollere Mensch wertloseren gegenüber höher gewertet werden muß und, mindestens geistig, herrschen soll. Es ist traurig, wenn das Genie der Masse unterliegt, statt daß diese sich ihm unterordnet. Worin liegt denn aber dieser höhere Wert desselben? Doch darin, daß er reicheren Lebens voll ist, als die anderen Menschen. Welches Lebens? Des Lebens, das sich in allen anderen ebenfalls darlebt, nur nicht so kraftvoll, eigenartig, schöpferisch, umfassend. Ist mithin dieses 
Leben, das in allen, mehr oder weniger ausgesprochen, lebt, in den übrigen nichts wert, dann auch nicht in dem Genius.

Die Voraussetzung für den höheren Wert des Genius ist gerade die, daß das Leben, wie es auch in jedem Manne aus dem Volke vorhanden ist, an sich etwas wert ist; $\mathrm{da} B$ die einzelne Menschenseele einen Selbstwert hat. So widerspricht sich Nietzsche selbst, wenn er die Persönlichkeit des Genius überhoch einschätzt und die bloß normalen Menschen, ja ganze große Gemeinschaften, aus welchen Genien hervorgehen, verachtet.

Der Genius gewinnt dadurch größeren Wert als andere, daß sein Mikrokosmus ein vollkommeneres Abbild des Makrokosmus ist, da $B$ er das Gemeinschafts- und Alleben wahrer und voller in sich aufnimmt und ausprägt. Der gemeinsame Grund, der sich im All, in der Menschheit, im individuellen Leben offenbart, fordert hier Beachtung. Er betätigt sich in jedem normalen Menschen auf eigenartige Weise. Die Folge ist, daß die menschlichen Einzelleben, mögen sie auch an sich einen relativ höheren Wert haben, als andere einzelne, dennoch der Ergänzung durch die Mehrheit derselben bedürfen.

Der Einzelne wird durch seine Eltern aus dem Ganzen der Menschheit geboren. So wird er von einer ihn umfassenden Mehrheit hervorgebracht, bedingt, getragen, erhalten. Insofern ist ihr Wert böher zu schätzen, als der jedes Einzelnen für sich.

Auch von dieser Seite her entspricht mithin der Altruismus allein der Wahrheit der Sache. Die Fülle des Lebens, welches auch die genialen Individuen erzeugt, ist demgemäß höher zu stellen, als selbst der bedeutendste Einzelne. Und zwar um so sicherer, je umfassender das Ganze ist. Ein Volk ist fast stets mehr wert, als ein von ihm erzeugter Genius; die Menscheit aber ohne alle Einschränkung, da sie das reichste Leben unzähliger Genien aus sich hervorbringt und in sich trägt. Der Wert jedes Einzellebens wird daher durch den des Gesamtlebens bedingt, das sich in den Einzelleben differenziert.

Schon von hier aus ist zu ersehen, daß das Alleben mit seinen Zielen die letzte Norm sowohl für das Einzelleben, als für das Gemeinschaftsleben sein muß. Je mehr beide das Wesen und Archiv f. Geschichte d. Philosophie. XVII. 1. 
dio Ziele des Gesamtlebens ausdrücken, desto höher steigt mithin ihr Wert. Dieser liegt zuletzt in der Erfüllung mit dem Geiste und Willen des Gesamtlebens. Religiös ausgedrückt: Der Wert des einzolnen Willens und des Willons der Gemeinschaft ist nach dem göttlichen Willen zu bemessen und za regeln.

Diesen Sachverhalt spiegelt das rechte Selbst- und Gemeingefïhl, sowie auf höchster Stufe das Welt- und Gottesgefühl wieder. Abor erst die fortschreitende Entwicklung des Geistes hebt diese Gefühle in ein zunehmend adäquates Bewußtsein. .

2. Nietzsches Begründung der Ethik auf das Prinzip. des Lebens.

Dies führt uns auf ein weiteres Verdienst, das sich Nietzsche um die Ethik erworben hat. Er gründet die Sittlichkeit nämlich auf das Prinzip des Lebens. Das ist ja überhaupt der Grundbegriff seiner Philosophie. Steht dem Buddhisten das Sterben, das Absterben und Aussterben am höchsten, so ist für ihn Ziel und Sinn menschlichen Verhaltens, daß ein jeder wahrhaft lebe und sich auslebe.

Mit diesem Hinweis auf das Leben selber hat er aber einen Weg gewiesen, den noch heute die meisten Ethiker vergeblich suchen. Wie unlebendig ist Kants Grundsatz der Sittlichkeit! Ich soll so handeln, daß die Maxime meines Willens als Prinzip für eine allgemeine Gesetzgebung gelten kann. Das ist doch rein formalistisch. Über die innere Beschaffenheit, über den Beweggrund des Wollens und Handelns ist damit nichts ausgesagt. Um die Regel meines eigenen Handelns zu erkennen, müßte ich zunächst die Gesetze des allgemeinen Handelns erkannt haben. Diese aber könnten sich wiederum, wenn ich das menschliche Gebiet nicht verlassen will, nur nach dem Zusammenwirken. der handelnden Einzelnen bestimmen. So kommt man aus dem Zirkel nicht heraus.

Dagegen ist das allseitige und vollständige Ausleben, was Nietzsche will, eben die Entfaltung, Ausbildung und Entwicklung der in der menschlichen Natur angelegten Eigenschaften und Funktionen. Hierin liegt ein naturgegebener, konkreter, faßbarer Stoff und Ausgangspunkt für jede Art menschlichen Handelns. 
Freilich wird sich dieses Ausleben des Einzellebens in seiner vollen Wahrheit nur bestimmen lassen, wenn man der Tatsache gerecht wird, daß dasselbe einem Gemeinschaftsleben, dem Menschheitsleben, dem Gesamtleben eingegliedert ist; daß es daher seine eigene volle Bestimmung. nur in seinem gliedlichen Zusammenhang mit dem zugehörigen Organismus verwirklichen kann. Nietzsches Fehler besteht hier mithin darin, daß er das "Leben", wonach er die Sittlichkeit normiert, nur als Einzelleben faßt oder doch betont.

Man hat also in der Ethik allerdings von der Selbstliebe und dem Werte eigenen Lebens auszugehen. Aber des Lebens, wie es zugleich vom Gesamtleben, vom Leben der Menschheit umschlossen ist. Nur so entsteht die Möglichkeit, beide Seiten gleichmäßig zu würdigen und dem Einzelnen die Stellung anzuweisen, die ihm in der Gemeinschaft zukommt.

\section{Die Autonomie der Sittlichkeit bei Nietzsche.}

Wenn Nietzsche die Sittlichkeit auf das Leben zurückführt, so ist daran auch dies als ein Fortschritt zu begrüßen, daß er sie dadurch aus den Wolken der Abstraktion herausreißt und wiederum dem Herzen des Menschen einpflanzt. Ein Gesetz, das nur jenseits des Menschen wäre, kann überhaupt kein Gesetz des Menschen sein. Auch wenn es die Sphäre des Menschen überragt, muß es doch in ihn eingehen, um für ihn gelten zu können. Die Sittlichkeit des Menschen kann zuletzt nur das Gesetz seines tiefsten Wesens sein.

Denkt man mithin Kants „radikales Böse" so wurzelhaft, daß es den Bestand der Wurzel selber ausmacht, dann ist überhaupt keine Möglichkeit, es daraus zu entfernen. Man hat es vielmehr als ein Gift zu betrachten, von welchem der Mensch bis in sein Innerstes hinein angesteckt ist. Das infizierte Wesen des Menschen selber aber kann nicht das Böse sein. Es liegt hier eine abnorme Zersetzung der Säfte vor, welche beseitigt werden muß. Ist der Mensch überhaupt nicht auf die Tugend angelegt, so kann er niemals tugendhaft werden. Ohne Bild geredet: Das Böse ist als eine Verletzung oder Verkehrung des Gesetzes und der Ordnung der menschlichen Bestimmung zu denken. 
Die Möglichkeit eines solchen scheinbaren Widerspruchs ist in der Eigenart des Menschen begründet, daß or zu dem, worauf er angelegt ist, erst in eincm komplizierteren Verlaufe werden kann. Er ist eben ein geschichtliches Wesen im spenifischen Sinne. Dies ist ein Merkmal, welches dem Tiere nicht zakommt. Hier andert sich in der Folge der Geschlechter das Individuum nicht wesentlich. Die Idee ihrer Gattung wird heute von den einzelnen zu ihr gehörigen Tierindividuen nicht nennenswert vollkommener erreicht, als in früheren Zeiten. Der Mensch aber.bildet einen "Übergang" von einem Reiche intelligenter Tierheit zu einem höheren Reiche. Er hat sich nachweisbar im Laufe der Jahrtausende im Ganzen zunehmend zu einer vollkommneren Art entwickelt. Er ist erst durch die Gesellschaft und Menschheit in ihrem geschichtlichen Leben zu seiner vollen Menschlichkeit .gelangt. Ganzer Mensch wird er überhaupt erst als Kulturmensch.

Sprache, Wissenschaft, Kunst, Sittlichkeit, Religion sind ebenso Erzeugnisse, wie Kennzeichen und Merkmale voller $\mathrm{Hu}$ manität. Sie sind im Laufe einer vieltausendjährigen Geschichte erworben und weiter ausgebildet. Unter diesem Gesichtspunkt kaun man den Naturmenschen, obwohl er doch durchgängig mindestens Sprache und Anfänge der Religion besitzt, nur seiner Anlage, aber nicht seiner Entwickelung nach als vollen Menschen bezeichnen. Ebensowenig, wie man diesen schon in dem Kinde anerkennt.

Demnach ist auch das Menschenleben nur. im Sinne des vollen Menschen in seiner lebendigen, bestimmungsgemäßen Eigenart zur Norm und Regel des sittlichen Rechtverhaltens zu erheben. Sobald man ihn dagegen, wie dies Nietzsche hier und da tut, vorwiegend nach seinen sinnlichen Naturtrieben, oder absehend von seiner ihm wesentlichen sozialen Beziehung und Bestimmtheit in Betracht zieht, wird man seiner Wahrheit nicht gerecht.

Nietzsche hat mithin soweit recht, als er die Wahrheit menschlichen Lebens als Gehalt, die Gesundheit und Kraft desselben als Form der Tugend.faßt. Doch verkennt.er, daß zur tiefsten Wahrheit des individuellen Lebens seine Erreugung aus dem und seine Bedingtheit durch das Gemeinschafts- und Gesamt- 
leben gehört; daß daher Wert und Zweck auch des seiner Wahrheit entsprechenden individuellen Lebens nicht allein vom Standpunkte des Einzellebens aus zu bestimmen sind. Die Entwicklung und Ausreifung desselben zur vollen Darstellung seines Wesens kann vielmehr nur in der Unterordnung unter das Gemeinschaftsund Gesamtleben Ziel oder Norm finden.

Sofern das Einzelleben von vornherein im Gesamtleben beschlossen liegt, ist mithin das Normale, daß sich die einzelnen Menschen mit zunehmender Entwicklung mit den höheren $Z_{w e c k e n}$ ihres übergeordneten Organismus erfüllen, dem sie angegliedert sind. $\mathrm{Daß}$ sie immer bewußtere und willigere Werkzeuge des größeren Ganzen werden, indem sie dessen Zwecke zu den ihrigen machen und dieselben in den eigenen Lebenszweck aufnehmen, oder vielmehr die eigenen Lebenszwecke immer vollständiger den $Z$ wecken des höheren Lebensganzen einfügen. So erreichen sie ihre eigenste Bestimmung; aber eben als eingeordnet in den sozialen Körper.

Wenn also Nietzsche die bis dahin nicht genügend berücksichtigte Seite der (wahren) Selbstliebe stark betont, so liegt hierin eine sachliche und geschichtliche Notwendigkeit für die Weiterentwicklung des höheren Denkens. Zugleich ist nicht zu verkennen, daß er dabei Darwins Gedanken stetiger Höherentwicklung durch den Kampf ums Dasein, jedoch hier nur innerhalb der Geschichte menschlicher Sittlichkeit, fruchtbar zu machen sucht. Tut er dies einseitig, übertreibend und überkühn, so pflegt sich geschichtlich das bis dahin unterschätzte Gewicht eines Faktors gerade in solcher Weise auszugleichen. Immerhin tritt er mutig an ein Problem heran, das man im Grunde bis jetzt noch kaum ernstlich anzufassen gewagt hat.

\section{Die sittliche Entwicklung des Menschen und der Menschheit.}

1. Der ursprüngliche Zustand vorwiegender Sinnlichkeit beim Naturmenschen.

Nietzsche sucht nämlich den Weg, welcher die Menschheit von einem vorwiegend sinnlichen Ausgangspunkte dem höheren Ideale entgegenführt. Es handelt sich um die Frage, wie der Natur- 
mensch zum Kulturmenschen, der vorgeschichtlicho zum geschichtlichen goworden ist. Und zwar steht für uns diese Entwicklang unter dem sittlichen Gesichtspunkte.

Fassen wir demnach die Entwicklung der Menschheit, sowcit sie uns angeht und wir ihrer habhaft worden können, nunmehr in konkreter Gestalt ins Auge.

Es ist kaum mehr darüber zu streiten, ob gewisse Sagen und Mythen, die zum Teil hochpoetisch und tiefreligiös sind und ewige Wahrheiten ausdrücken, wörtlich zu nelımen sind und in diesem Sinne die Entwicklungsgeschichte der Sittlichkeit unbedingt entscheiden. Dann müßte schon dem ersten Menschen eine, wenn auch noch nicht völlig vollendete, sittliche Vollkommenheit, statt sittlicher Anlage, zugeschrieben werden. Auch Rousseau meint, daß im wesentlichen erst die Entwicklung des Naturmenschen zum Kulturmenschen dessen sittliche Unvollkommenheit verschuldet habe.

Wir wissen bis jetzt nicht viel von dem vorgeschichtlichen Menschen. Soviel aber darf als ausgemacht gelten, daß der Mensch nicht gleich als Kulturmensch auf der Erde auftritt. Er hat sich vielmehr jedes Stück seiner Kultur in heißem Kampfe mit der Natur und mit seiner tierischen, menschlichen und sonstigen Umgebung, auch in innerem Streite mit sich selbst, erringen müssen. Dabei kommt Darwins Hypothese, der den Menschen direkt oder indirekt von einer besonders vollkommenen Tiergattung abstammen läßt, hier garnicht unmittelbar in Frage. Dies würde unter unserem Gesichtspunkte nur ein Streit um Worte sein.

Hat der Mensch sich die ganze Natur in stetigem Werdegang unterwerfen müssen, so fehlte ihm anfangs notwendig alle Erfahrung, die ihm erst aus diesem Verkehr mit der Natur und der Arbeit an ihr erwạchsen konnte. Auch Mäßigung und Selbstbeherrschung konnte nicht von selbst kommen. Der Mensch mußte seine eigene Natur erst bezwingen, ihre niederen Triebe den höheren unterordnen lernen. Er war mit einem Worte ein kraftvolles, gesundes, aber unreifes Wesen, das erst allmählich aus der Fläche in die Tiefe, aus den sinnlichen in die geistigen Interessen hineinwachsen konnte. 
Daher war er auf diesem Wege notwendig auch in seinen einzelnen Entschlüssen und Taten maunigfachen intellektuellen Irrtümern, sowie Irrungen in seinen Werturteilen ausgesetzt. So mußte es wiederholt zu Handlungen kommen, in welchen er z. B., wenigstens für den Augenblick, sinnliche Zweeke höheren Zwecken überordnete. Denn gerade diese Ordnung entsprach der zunächst rorwiegend entwickelten sinnlichen Seite seines Tesens. Dagegen widersprach sie allerdings seiner höheren Bestimmung, deren Terwirklichung seine Aưgabe für die Zukunft war.

In diesem objektiven Sinne ist für ihn schon jetzt die "Sünde" rorhanden. Da aver des Menschen sittliches BewuBtsein noch nicht hinreichend ausgereift war, so fehlte ihm das BewuBtsein auch der Sünde. Der Nlensch war trotz allem noch frei von eigentlicher Schuld $^{*}$, d. h. von der mit bewußter Absicht begangenen Sünde. Erst für den rückwärts blickenden Jenschen, der sich zugleich seiner in der Zukunft liegenden Bestimmung berußt wird, für den sich also die punttuelle Gegenwart nach beiden Seiten hin organisch erweitert hat, und der nun seinen gegenwärtigen Zustand oder seine Handlung an diesem Ideale mißt, kann das Bewnßtsein der Sünde entstehen. Das Innewerden jener höheren Beziehung konnte sich erst nach und nach klären, befestigen und kräftigen.

Lm dies einzusehen, muB man vor allem bedenken, daß der Jaturmensch, auch heute noch, mehr oder weniger ein Kind des Augenblicks ist. Er konnte daher anfangs garnicht anders; als durch Impulse bestimmt werden. Denn die Empfindungen, mit welchen alle Erfahrung beginnt, sind als solche Augenblickserregungen. Denken und Urteilen aber kommt anfangs immer erst, auf Grund ron Erfahrungen, eine Strecke hinter den Empindungen und binter den Handlungen her, welche durch die sinnlichen Triebe, zunächst unwillkürlich, ausgelöst rerden. Tem hätte also der Mensch in jenem Zustande überbaupt folgen können und sollen, als seinem unmittelbaren Begehren?! Seine, zunächst unreife, Handlungsweise konnte sómit nur auf Grund wiederbolter und längerer Erfahrung Klarheit, Festigkeit und Dauer gewinnen.

Die Erscheinungen, die sich unter dem Gesichtspunkte entwickelterer Sittlichkeit als Selbstsucht und Sünde darstellen, 
sind demnach zunächst nur als Symptome menschlicher Unreife aufzufassen.

Aus allodem orgibt sich also Recht und Aulah, wie noch jetzt jedem, zunächst unreifen, Kinde, so der. Menschheit, auch in sittlichor Hinsicht, ein allmähliches Ausreifen zuzuschreiben. Jedes Kind ist von Hause aus weit überwiegend „Egoist". Seine zunehmende Entselbstung kann erst ein. Produkt der Entwicklung sein. Aber es ist schuldlos und weiß noch nichts von bew ußter Sünde. Erst wenn das Kind in abnormer Dauer auf dem Standpunkte der Kindheit zurückbleibt, darf dies als Krankheit gelten. Aber ganz normalerweise geht erst aus der Unreife des Kindes, durch das stürmische Begehren des Jünglings hindurch, eine allmähliche Ausbildung der höheren, geistigen Interessen hervor.

Einen derartigen Entwicklungsgang muß auch der Fortschritt der Menschheit im großen in seiner Weise aufweisen. Erst ein abnormes Stehenbleiben derselben auf vormenschlichem, untermenschlichem Standpunkte darf auch hier, im besonderen auf sittlichem Gebiete, als "Atavismus" gelten. Auch der Naturmensch vermochte sich erst nach und nach von einem einigermaßen tierähnlichen, kindlichen Egoismus, zum bewußten Altruismus zu erheben.

Hieraus erwächst, wenigstens im allgemeinen, die Berechtigung, die Art des Naturmenschen und der menschheitlichen Entwicklung durch den Werdegang des Menschenkindes zu beleuchten, wie wir ihn noch jetzt in der Kinderstube beobachten können.

Die ethnologischen Forschungen der letzten hundert Jahre haben, bei aller Lückenhaftigkeit, hinreichendes Material erbracht, um die geschichtlichen Anfänge wichtiger Kulturvölker, wenigstens in ihren Grundzügen, zu erkennen. Ich nenne nur die Römer, die Griechen, die Inder, die Chinesen, die Perser, die alten Deutschen. Auch können wir diese Kenntnisse durch das Verhalten mancher noch jetzt nicht oder nur wenig kultivierten sogenannten "wilden“ Völker ergänzen. So lassen sich, bei allen Vorbehalten im einzelnen, bedeutsame Punkte ihrer durchgängigen Entwicklung, auch in sittlicher Hinsicht, geschichtlich feststellen oder doch mit hinreichender Sicherheit erschließen. Gerade hier hat Nietzsche nun schätzbare Beiträge:zur Aufhellung mancher dunkeln Stellen geliefert. 
Indessen kann ich an diesem Orte nur auf einige Hauptgedanken aus seinen für unsern Gegenstand grundlegenden Schriften : "Jenseits von Gut und Böse" und „Genealogie der Moral" eingehen. Nur muß man nach obigem von vornherein gewärtig sein, da $\beta$ : der enthusiastische Prophet des Individualismus nur diejenige Seite der sittlichen Entwicklung sielt, die ihren Anstoß aus dem Egoismus der Individuen in ihrem Kampfe mit einander empfängt, dagegen für die stilleren, verborgeneren Bewegungen, die dieser Selbstsucht in der Tiefe des Menschenherzens widerstreiten, keinen unbefangenen Blick hat. So wird die Entstehung der sittlichen Vorstellungen unter dem äußeren, objektiven Gesichtspunkte meist richtig, wenn auch zu grell beleuchtet. Hingegen der tiefere Vollzug der Entwickelung des Subjekts selber auf Anlaß jener egoistischen Zusammenstöße findet, wenigstens nach seiner altruistischen Seite, keine gerechte Würdigung. Daher die cynische Verzerrung so mancher Vorgänge des Seelenlebens zur Karikatur. Daher die einseitige Herleitung der "selbstlosen" Liebe aus bloßer Schwäche und Unvermögen, ernstester Selbstïberwindungen in Reue und Buße aus nach innen geschlagener Rache und asketischer Grausamkeit, unverfälschter Demut aus verstecktem Ressentiment einer Sklavenseele.

Doch liegt mir hier mehr daran, die Grundzüge der Entstehung sittlicher Vorstellungen im allgemeinen zu skizzieren, als dieselben in allen einzelnen Arten der Seelenbewegungen nachzuweisen. Ich lege nur den Ton darauf, wie sehr die von Nietzsche vernachlässigte altruistische Seite des subjektiven Vorgangs der Hervorkehrung bedarf. Denn allein in der Tiefe des Subjekts kann jener zarte Gesinnungswandel vor sich gehen, der aus dem Naturmenschen den Kulturmenschen, aus dem unreifen den gereiften, aus dem egoistischen den selbstlosen, aus dem unsittlichen oder sittlich unentwickelten den vollsittlichen Menschen hervorbringt.

\section{Die „Herrenmoral“.}

Dem Dargelegten gemäß, herrscht in den ersten Anfängen menschlichen Lebens naturgemäß das Recht des Stärkeren. Und 
zwar im physischon und seelischen Betracht. Dabei ist es selbstverständlich, daß jeder dic ihn mitgegebene Überlegenheit der größeren Kraft oder Iist in weitgehendem Maße anwondet. Mit welcher Harmlosigkeit macht selbst noch ein Odysseus von letzterer Gobrauch! Hierin hat Nietzsche sicherlich recht.

Wenn sich Kinder miteinander um Güter oder Genüsse streiten, so gewinnt auch heute noch der Kräftigere, Willensstärkere oder Klügere die Oberhand und bedient sich seines Vorteils mit fast völliger Rücksichtslosigkeit. Auch erwäge man, daß Kraft und Wert des leiblichen und seelischen Lebens in den ursprünglichen Verhältnissen der Menschheit noch fast gänzlich parallel liefen, daß demnach die körperlich Kraftvolleren auch die geistig Tüchtigeren zu sein pflegten. Wenn man ganze Völker nimmt, so ist dies ja bis zu einem gewissen Grade auch jetzt noch der Fall. Die Kehrseite davon ist die, daß leibliche und sittliche Entartung bei den Völkerschaften in Wechselwirkung zu stehen pflegen.

So vergevaltigt in der Regel das tüchtigere Volk im Kampt ums Dasein das untüchtigere und gibt ihm gewisse Gesetze. Unleugbar mußte auf diese $W$ eise die herrschende Rasse der niederen auch mancherlei Anschauungen von der rechten und verkehrten Art zu leben aufprägen. Sicherlich schätzte sich der Stärkere als den Vorzüglichen, Besseren, sah den Besiegten, den Schwäcberen, als den Minderwertigen an und behandelte ihn dementsprechend.

So weist. denn Nietzsche mit Recht darauf hin, daß sich Spuren einer solchen "Herrenmoral" noch jetzt erhalten haben und sich auch sprachlich nachweisen lassen. Dies gilt natürlich nicht nur von Völkern, von denen das eine das andere besiegt und unterwirft, sondern auch von Gruppen, Stämmen und Klassen innerhalb eires Volkes, unter denen die einen den anderen übergeordnet sind und sie mehr oder weniger beherrschen und bestimmen.

Nur ein aristokratisches Augenmaß kann auf deutschem Gebiete den "schlichten" Mann in einen "schlechten" umgewandelt und auf griechischem den mit Mühe beladenen (poneros, mochtheros) als „schlecht", „böse" mit dem Nebengedanken des „Unglücklichen" gestempelt haben. Ähnlich wird der Furchtsame und Feige als 
schwach, schlecht und unglücklich geachtet, denn zu allen diesen Bedeutungen kommt das Wort "deilos".

Dagegen genießt der Held, welcher den Schwachen überwältigt, in erster Linie Achtung und Bewunderung. Vor allem bei seinesgleichen. Ja seine Stärke, wenn auch nicht deren Anwendung, macht allgemeinen Eindruck und erzwingt, selbst bei den Unterdrückten, freilich widerwilligen, Respekt. Stärke, Geschicklichkeit und List stehen bei unreifen Menschen, zumal im Naturzustande der Menschheit, aber auch noch in späteren Perioden, fast einzig im Werte, während den Schwachen, welcher unterliegt, den Gutmütigen, der sich etwas gefallen läßt, Verachtung und Schimpf trifft. Nur dem Sieger wird Großmut und Hochherzigkeit gegenüber dem Besiegten zugute gerechnet. Insofern jene Stärke freilich verderblich wirkt, tritt bei den Unterdrückten, wie wir sogleich sehen werden, noch ein anderes Gefühl hinzu.

Dem Vergewaltiger als solchem liegt nun, unter gewissen Einschränkungen und bis zu einem gewissen Maße, das Schuldgefühl zunächst fern. Zumal bei einer Vergewaltigung von Fremden. Jene wird mit gänzlicher Harmlosigkeit, ohne eigentliche Bosheit, geübt. Erst engere Zusammengehörigkeit von Menschen läßt zartere Gefühle aufkommen. Dies Zwingen und Vernichten geschieht um so naiver, als der Vergewaltiger, je unreifer er ist, destoweniger die Folgen seines Tuns übersieht.

Ein Tier tötet das andere, wenn man von unmittelbarster Gattungszugehörigkeit absieht, ohne die geringsten Bedenken. Die zärtliche Katzenmutter bringt ihrer Aufzucht, mit dem Gefühle stolzer, fast humoristischer Überlegenheit, junge Mäuse und Vögel, um sie mit diesen ihr grausames Spiel treiben zu lassen. Der Held der Urzeit kann, wie schon aus seinen Taten zu schließen ist, nicht viel anders empfunden haben, wenn er einen Gegner niederwarf oder einen Gefangenen marterte und qualvoll tötete. Er gab leicht genug sein eigenes Leben preis. Noch leichter aber das Leben derer, welche ihm im Wege standen, sofern sie ihm nicht die Spitze bieten konnten.

Ein Beispiel dafür, wie wenig Skrupel sich auch ein christliches Volk aus grausamer Ausrottung eines anderen macht, hat 
uns in neuester Zoit der Krieg dor Engländer gegen die Buren geboten.

Sovicl ist wahr an Nietzsches Herrenmoral.

\section{Nietzsches Sklavenmoral.}

Aber auch Nietzsches Ableitung der "Sklavenmoral" enthält manches Wahre. Auch hier wird man das von ihm Gefundene nicht ohne weiteres verwerfen dürfen, vielmehr die Grenzen abstecken müssen, innerhalb deren es Geltung hat. Es ist eine alte Erfahrung: wer nicht selbst gelitten, hat mit anderen kein Mitleid. Das Kind, wie das Tier, ist nur deshalb oft so hartherzig und grausam, weil es den Leidenden nicht sich selber gleichsetzt. Erst wer gelitten hat, lernt das Mitleid schätzen. Eigener Schaden macht zunächst klug und dann auch mitfühlend. Das Mitleid. mit Fremden wenigstens wird im Kreise der Unterdrückten oder Leidenden seine erste Schätzung und Würdigung gefunden haben.

Allerdings kennt die Liebe der Mutter, selbst schon der Tiermutter, ein gewisses Mitgefübl mit den von ihr Geborenen. Denn die Kinder bedeuten schon. leiblich die Errweiterung ihres eigenen Selbstes. Doch ist zu beachten, daß auch dieses Mitleid teilweise auf vorhergegangenen eigenen Leiderfahrungen ruht.

Wiederum ist selbst das elterliche Mitgefühl nur relativ selbstlos. Es hat, vor allem auf seiten des Vaters, seine Grenzen, zumal bei Naturvölkern. Dabei sehe ich davon ab, ob sich, auf frühester Stufe, der Vater das Kind überhaupt zugerechnet hat.

Sogar die Mutterliebe hält nicht immer Stich. Sie pflegt bei den Tieren nur solange zu bestehen, als die Jungen durch das dringendste Bedürfnis an ihreSäugerin und Beschützerin gekettet sind. Umgekehrt ist ja in gewissem Maße auch die Mutter, schon körperlich, auf ihre Säuglinge angewiesen.

Allerdings beginnt die Mutterliebe bei den Naturvölkern dauerhafter zu werden und sich zu vergeistigen. Die Hilflosigkeit des Menschenkindes ist übrigens auch weit größer als die der Tierjungen.

Sobald sich's aber um Fremde handelt, versagt das Mitgefühl leicht, zumal bei Menschen niedrigerer Stufe. Hier haben die 
Vorstellungen der furchtbaren Leiden, die die Naturmenschen einander zufügen, nicht genug Wärme und persönliches Interesse, um von den Urhebern derselben auch nur in ihrer ganzen Bitterkeit begriffen zu werden. Es sind für sie Ereignisse, bei denen sie vorwiegend die eigene Leidlosigkeit, sowie das Gefühl ihrer Stärke und Überlegenheit genießen. Sie wenden jene grausamen Bilder nicht oder nur ganz entfernt auf die eigene Person an.

Doch hat Nietzsche hier soweit unrecht, als er von der natürlichen Sympathie der Menschen für die absieht, die ihnen gänzlich als ihresgleichen erscheinen, weil sie mit ihnen durch engste Blutsverwandtschaft verbunden sind. Sonst könnte er das Mitleid nicht ausschließlich aus der Empfindung der Leidenden herleiten.

Noch schlimmer aber ist es, wenn er das Mitgefühl, wo es sich auch findet, möglichst durch selbstsïchtige Motive verunreinigt und im Grunde überhaupt nur an Egoismus glaubt. Hier führen ibn die Konsequenzen seines einseitig individualistischen Prinzips über die Grenzen aller Naturwahrheit hinaus. Richtig ist nur, daß das Mitleid oft mit anderen selbstsüchtigen. Motiven versetzt und dadurch getrübt und unlauter wird. Dennoch sollte man nicht nötig haben zu versichern, daß es nicht nur egoistische Triebe, sondern auch altruistische Neigungen, daß es echtes Mitgefühl gibt. Und zwar bei den edelsten Geistern, deren altruistischer Wein schon aus dem egoistischen Most herausgeläutert ist, am meisten. Es ist eine Blasphemie, alles Mitleid aus Willens- und Nervenschwïche herzuleiten. An Wahnsinn aber grenzt es, gar das ganze Christentum aus weichlicher Nervenschwäche und heimtückischer Herrschsucht zu erklären und es als den raffiniertesten Sklavenaufstand darzustellen. Damit wird das Göttliche im Menschen besudelt, und die lauterste Blüte gottähnlicher Nächstenliebe und Humanität in den Staub getreten.

Die Fähigkeit des Mitgefühls ist von Anfang an im Menschenherzen vorhanden. Denn sie ist ja schon in dem natürlichen leiblich-seelischen Instinkte der Gleichartigkeit begrïndet, den selbst das Tier in einem gewissen Grade besitzt. Wäre der sich hierauf gründende Altruismus nicht von vornherein im Menschen angelegt; gehörte er nicht, wie ich gezeigt habe, mit zur Wahrheit des echten 
Monschentums, so würde sich wahres Mitleid überhaupt niemals ontwickeln können. So sehen wir das Mitgefühl im Verkehr der Menschen miteinander zwar erst nur im stillen wirken, nach und nach aber, wenigstens im großen, je länger, je mehr vorwiegend.

Das aber ist freilich wahr, daß sich dieses Mitgefühl bei dem Urmenschen erst allmählich aus vorwiegendem Egoismus herausarbeiten mußte und nur als späte Frucht höchster Humanität in vollkommnerer Gestalt auftritt. Der Altruismus betätigt sich, wie wir sahen, anfangs nur in engsten Grenzen, entfernt sich erst langsam von dem reinen Naturboden und erweitert seinen Spielraum dann auf zunehmend umfassendere Kreise. Es bedurfte jedoch langer Zeiträume und harter Anstöße, um die Schale der Borniertheit des Kopfes und Herzens zu sprengen und die fruchtbaren Ströme der Liebe auf weitere Gefilde hinüberzuleiten.

Wollen wir ein Beispiel aus der jüngsten Zeit dafür, wie sich eine derartige Vertiefung und Verfeinerung der sittlichen Anschauungen vollzieht, so brauchen wir nur an die soziale Frage zu denken. Noch vor 40 Jahren waren sich nur ganz Vereinzelte bewußt geworden, daß die Gesellschaft verpflichtet sei, der durch die Entwicklung der Technik entstandenen Arbeits- und Nahrungslosigkeit der unteren Klassen abzuhelfen. ${ }^{1}$ ) Nunmehr ist diese Pflicht in jedermanns Bewußtsein übergegangen. Aber wie ist das gekommen? Nicht etwa so, daß dies Pflichtbewußtsein von selbst, aus innerer Freiheit, erwacht wäre. Nein! Der handfeste Sturm der Sozialdemokraten und die von dieser Seite für die Gesellschaft drohende Gefahr hat jene altruistische Einsicht zuerst in den besten Herzen wachgerufen. Andererseits aber wäre es "höchst verkehrt, das gesamte Erwachen des sittlichen Bewußtseins in dieser Hinsicht aus $Z$ wang und Furcht zu erklären. Das waren nur die äußeren Anstöße, die sodann eine Entwicklung des sittlichen Gefühls und daraufhin eine Weiterbildung der sittlichen Anschauungen veranlaßten. So entwickelten sich diese Regungen wahrer Nächstenliebe wenigstens bei allen besseren Menschen, während freilich bei vielen anderen die Angst das herrschende

1) Von den staatlichen Behörden sehe ich dabei ab. 
Motiv geblieben sein mag oder doch die Lauterkeit der Gesinnung durch anderweitige Beweggründe teilweise getrübt wurde.

\section{Das „Böse“.}

Wir werden nun sogleich dazu übergehen, zu untersuchen, auf welche Weise der Mensch aus einem Egoisten zum Altruisten wurde. Nur einem wichtigen Begriff wollen wir uns zuvor noch ausdrücklich zuwenden, welchen Nietzsche ebenfalls auf die sogenannte Sklavenmoral zurückführt. Es handelt sich um das eigentlich sogenannte Böse. Es fragt sich, wie man dies zuerst in seiner Verwerflichkeit empfinden lernte.

Den richtigen Weg scheint schon die Tatsache zu weisen, daß es das gerade Gegenteil des Mitgefühls darstellt. Nietzsche hat gewiß darin recht: es ist eine andere Gesinnung, wenn der mutige, starke und stolze Sieger auf das Schwache und Furchtsame als auf etwas Gemeines, Unvornehmes, Feiges und in diesem Sinne Verächtliches herabblickt und es als "schlecht" bezeichnet, und wenn umgekehrt die Schwachen die Gesinnung solcher Leute deuten, von denen sie rücksichtslos geschädigt, verderbt, vernichtet werden, und dies Verfahren „böse“ nennen. Es ist daher in der Tat einleuchtend, wo wir die ersten Erfahrungen des eigentlich Bösen zu suchen haben werden. Das Böse im ethischen Sinne wurdé nicht zuerst von denen mit tieferem Eindruck empfunden, welche es übten, sondern die durch dasselbe litten. Man wird sich. deshalb nicht wundern, wenn der entsprechende Begriff und das Wort dafür in erster Linie von den Unterdrückten geprägt worden ist.

Während also Starken und Mutigen, welche der anderen Herr zu werden vermochten, eben diese Herrengesinnung als "gut" erschien, erkannten die Unterdrückten und. Geknechteten hierin gerade das "Böse", hingegen das wahre Rechtverhalten des Menschen gegen den Nächsten, mithin das "Gute“, vorwiegend in dem Gegenstück des durch die Unterdrücker erfahrenen Bösen.

Man sollte diese wichtigen Punkte an Nietzsches Herren- und Sklavenmoral nicht in Bausch und Bogen bestreiten; zumal ja doch früher oder später dasjenige daran anerkannt werden muß, 
was der Wahrheit entspricht. Erst so crwirbt man das volle Recht dazu, dio mancherloi verkehrten und verderblichen Folgerungen abzuwohren, dic der geistvolle Fanatiker daran knüpft, und um derentwillen man abgeneigt ist, auch die von ihm gefundenen Wahrheiten einzurïumen.

So ist es richtig, wenn Nietzsche betont, daß die heimtückische, aus Ressentiment, aus zurückgetretenem Rachegefühl entstehende Ranküne der Unterdrückten oft viel boshafter ist, als. die ruicksichtslose, dreiste und freche Vergewaltigung. Die Dauer des ungestillten Rachedurstes macht in der Tat die Stimmung erst wahrhaft krankhaft, giftig und eingefleischt, während der Sieger nach erreichtem Ziele sich über das Vergangene hinwegsetzt und auch sein Zorn keinen Anlaß hat, in bitteren $\mathrm{Haß}$ auszuarten.

Sehr verkehrt ist es nun aber, wenn Nietzsche das Böse eigentlich nur in der Rachsucht des Unterdrückten finden will und ihr gegenüber das lieblose, ja oft unmenschliche Niederwerfen und Vernichten der Schwächeren von seiten der Stärkeren im Grunde „gut“ und nur durch die feige Niedertracht der Unterdrückten fülschlich zum "Bösen" umgewertet findet. Freche Vergewaltigung kann selbst den Edelsten vorübergehend zur Rachsucht entflammen, und der Grad der Gewissenlosigkeit im Verüben von Mord, Brand und Schändung ist wahrlich nicht das Maß für wahren Adel der Gesinnung, trotz des blutigen Cesare Borgia. Hier wird denn doch die Form der Tugend gründlich mit ihrem bereits oben angegebenen, echten Gehalt verwechselt.

Der Grundfehler unseres Philosophen besteht auch hier wieder darin, daß er die von ihm zuerst gesehene und überscharf beleuchtete eine Seite der Wahrheit für die ganze Wahrheit nimmt. Infólgedessen -meint er kurzsichtigerweise, durch seine Erwägungen die Objektivität und den ewigen Wert der Vorstellungen „gut" und „böse" aufzuheben, wähnt die tiefsten Grundanschauungen der Sittlichkeit zufälligen, ja niedrigen Motiven entsprungen und glaubt sie daher einer radikalen Umwertung bedürftig. So gerät er hier wirklich in die Gefahr, die Gewalt des Stärkeren als die allein berechtigte Maxime für das Verhalten der Menschen untereinander hinzustellen, der "blonden Bestie", ein unverdientes Denkmal zu 
setzen und den Grundsatz jener zucht- und gottlosen Assassinen; nichts sei böse und alles erlaubt, als die unübersteigbare Höhe der Weisheit auszugeben.

Es ist indessen auffallend, wie nachdenkliche und gerechte. Leute hier haben den echten Nietzsche finden wollen, während dies doch nur die Entartung seiner kraftvollen, aber fanatischen Einseitigkeit bedeutet. Es wird eben immer noch das Grundstreben des Philosophen nicht verstanden, der selbst erklärt hat, daß man es mit seinen, zumal in der Polemik ausgestoßenen, Worten nicht genau nehmen dürfe. Dergleichen waren Bombenschläge, mit welchen er denjenigen vergalt, die in allem, was er sagte, weil es einseitig und teilweise gefährlich war, das Wahre, Neue, Notwendige blind verkannten und nur Gift und Lüge darin sahen. Denen gegenüber, welche in ihm den Satan witterten, war er stolz darauf, sich als solcher zu gerieren.

Wenn er die Naturkraft überschätzt, so liegt der Grund vor allem darin, dass er allein in der vollen Gesundung der erkrankten Zeit das Heil erblickt. Dies führt ihn nun aber auch dazu, die Grenze zwischen dem sinnlichen und sittlichen Wesen des Menschen zu verschieben und das Rechtverhalten desselben hier und da nur im Geltendmachen seiner Überlegenheit an Körperkraft und Willen zu erblicken, ohne viel nach der Qualität und der sozialen Beziehung dieses Willens zu fragen. Und doch macht, wie wir sahen, gerade diese erst den wahren Wert des Menschen aus. Jener Fehlgriff Nietzsches geht eben wieder deutlich auf seine einseitig individualistische Grundanschauung zurück. Denn wer keine Gemeinschaft kennt, nach welcher sich das Individuum zu richten hat, sondern umgekehrt jene durchaus in den Dienst des Einzelnen stellt, der kann das Ideal der Sittlichkeit nur in rücksichtslosem Egoismus finden. Sind jene Voraussetzungen des Philosophen richtig, dann verdient es pure Anerkennung (sofern dafür überhaupt Raum bleibt), wenn das Individuum die Wahrheit der Sache mit aller ihm zu Gebote stehenden Macht vertritt. Diese Wahrheit aber kann dann in nichts anderem bestehen, als in der unbedingten Überlegenheit der Persönlichkeit. Denn die Kraft ist die rechte Art aller Tugend. Es fragt sich nur, worein die Tagend zu setzen Archiv f. Goschichto d. Philosophie. XVII. 1. 
ist. Ihr kommt das wahre Recht des Stärkeren zu. So ist ibr zuletzt der Sieg gesichert. Nur derjenige wird unmittelbar oder mittelbar Sieger bleiben, der als Individuum die wahrheitsgemäße Stellung zum Ganzen, zur Gemeinschaft, zur Menschheit innehält. Jenn aus der Wahrheit fließt die Kraft. Nur entspricht gerade die Einordnung und Unterordnung des Einzelnen gegenüber dem Ganzen der Wahrheit der Sache. So behält die Selbstliebe nur Recht in stetiger Beziehung auf die Nächstenliebe; der Egoismus ist durch den Altruismus zu bändigen, zu regeln und einzuschränken.

\section{Das Gedächtnis die Voraussetzung der Sittlichkeit.}

Indessen ist nunmehr, zu schärferer Bestimmung des Sachverhalts, die allgemeine seelische Lage noch näher zu prüfen, in welcher sich der Naturmensch befand, als in seiner Seele die ersten sittlichen Vorstellungen entsprangen. Dann wird sich zeigen, daß, so verkehrt Nietzsches grundsätzliche Auffassung vom Wesen der Sittlichkeit sein mag, dennoch von ihm die Richtung des Weges im großen und ganzen richtig angegeben ist, auf welchem die Menschheit zu Kultur und Sittlichkeit emporstieg.

Wir greifen auf die oben gemachte Bemerkung zurück, daß der kindliche Urmensch, wie noch heute das Kind, naturgemäB zunächst für den Augenhlick lebt und sich erst langsam eine $\mathrm{Zu}$ kunft im volleren Sinne erwirbt. Es gibt ja selbst in unseren höheren Kulturen noch Menschen von so leichtem Sinn, daß es ihnen an Gedächtnis, wenigstens in sittlicher Beziehung, fast gänzlich mangelt. Selbst andauernde Erziehung, Zucht, Einschränkung und Strafe vermögen dem oft schwer oder nur teilweise abzuhelfen. Dieses Leichtnehmen ist es, vermöge dessen dem Tiere, dem Kinde und Naturmenschen kaum ein Augenblick der Lebensfreude entgeht, wodurch so schnell auch alles Schwere vergessen wird. In Schranken gehalten, bleibt solch leichter Sinn selbst für den entwickelten Menschen eine herrliche Gabe.

Andererseits aber wirkt er im Verkehr der Menschen untereinander in vielen Hinsichten äußerst schädlich. Das impulsiv gegebene Versprechen wird nicht gehalten, auch der gute Vorsatz 
nicht erfüllt, sobald die Neigung vorüber ist, die dazu trieb. Das schwerste Vergehen wird ohne Spur von Reue vergessen, als wäre es nie gewesen. Kein fester Plan wird für die Zukunft gemacht. Daher stürzen die Leichtsinnigen oft blindlings ins Verderben and ziehen viele andere mit.

So vermochten denn gewisse Sitten, die sich unter den Naturmenschen zu bilden begannen, erst allmählich größere Dauer zu erlangen. Von einem Sichverpflichtetfühlen, welches nicht durch unmittelbare Naturtriebe eingegeben und festgehalten wurde, konnte erst nach und nach die Rede sein. Eigentliche Gerechtigkeit gab es zunächst gar nicht, oder doch nur in sehr eingeschränktem Maße und instinktiver Art. Jedenfalls pflegte sie nur soweit geübt zu werden, als es dem, welcher die Macht besaß, augenblicklich anstand.

Uim auch nur die seelische Unterlage für eine eigentliche Sittlichkeit zu schaffen, dazu war also vor allem nötig, daß der. Mensch nicht mehr bloß Kind des Augenblicks blieb, sondern eine Vergangenheit und vor allem auch eine Zukunft bekam. Mit anderen Worten: zunächst mußte sich das Gedächtnis kräftiger entwickeln. Ohne Gedächtnis kein Gewissen, kein Pflichtgefühl, kein Schuldbewußtsein, keine Sünde u.s.w.

Alle diese Seelenbewegungen waren unmöglich, so lange der Erinnerung die hinreichende Dauer fehlte. Denn damit mangelte auch dem Verstande die Möglichkeit rechter Überlegung und dem Streben die nötige Zuverlässigkeit.

Es handelt sich mithin nunmehr um die Art und Weise, wie dem menschlichen Gedächtnis in Urzeiten, vor allem sofern es sich auf den Verkehr der Menschen untereinander bezog, am wirksamsten Dauer verschafft sein mag.

Bei Tieren, bei Kindern, bei niedrigstehenden Menschenklassen der jetzigen und früherer Zeiten pflegt das Gedächtnis am lebhaftesten körperliche "„Denkzettel ", Schmerzen und Schädigungen festzuhalten. Welches Kind gerät, selbst in unserer Kulturwelt und unter den Höchstgebildeten, ohne Schläge und Züchtigungen!

Die ursprünglichen Menschenhorden schädigen und vernichten sich gegenseitig schonungslos, zumal sofern sie einander fremd und 
feind sind. Aber nun entsteht bald die Rache in der Form der Blutrache. Denn die Urmenschen tragen ihre Schädigungen einander gar sehr nach. Sie warten beharrlich die günstige Gelegenheit der Vergeltung ab. Ja sie machen aus dieser eine religiöse Pflicht und verleihen der Rachbegier dadurch noch kraftvollere Dauer.

Zeigt sich doch auch in dieser Rache nicht nur die Begierde, den Mut zu kühlen, sondern auch das Gerechtigkeitsgefühl in ursprünglichster Form, welches, zumal unverdiente, Schädigung weder an sich noch an anderen dulden will. Der Kreis für die Ausübung dieser Gerechtigkeit ist dabei freilich noch eng und egoistisch abgesteckt. Genug: unzählige, kaum je aussterbende Fehden sind die Folgen dieser Blutrache, durch welche sich die streitenden Parteien oft bis zu gänzlichem Erlöschen bekämpfen.

In derartigen Kriegen verfuhr man oft auf das grausamste wider die Gegner mit Martern und Tötungen. Es erklärt sich dies aus verschiedenen Gründen. Einmal hatte das Sterben für die damaligen Menschen noch bei weitem nicht die Wichtigkeit, wie später. Vor allem, weil eben der Sinn für die Zukunft noch weniger entwickelt war. Doch fühlte man auch die Qualen und Schmerzen, die man sich gegenseitig zufügte, noch nicht so heftig, wie die Kulturmenschen.

Diese Kriege nun, in welchen man mit andauernder Aufmerk-, samkeit des Gegners Blößen belauerte und seine schwachen Stunden abwartete, mußten das Gedächtnis ungemein üben und kräftigen. Sodann aber sind es Marter und Tod, durch die man sich in jenen Fehden auf das nachdrücklichste ein Gedächtnis schuf. Natürlich wurden die zunächst kriegerischen. Gewohnheiten der Schädigung weiter unwillkürlich auf das Leben im Frieden, welches nur eine Păựe zwischen Kriegsfahrten zu bedeuten pflegte, übertragen.

6. Entstehung sittlicher Begriffe aus dem Schuldverhältnis.

Besonders wird dies von solchen Gelegenheiten gelten, bei welchen man im ursprünglichsten Verkehr gegenseitige Schädigungen wettzumachen suchte; z. B. wenn gegebene Versprechungen nicht 
gehalten, und wenn gar, in etwas späteren Zeiten, förmliche Verträge gebrochen wurden.

Falls eine Gegenleistung, auf die einer Anspruch zu haben glaubte, nicht gewährt ward, so schritt der Erzürnte, anfangs gewiß oft ohne weiteres, zur Vernichtung des Schuldners. Ein gewisses Gleichgewicht der Macht aber und der eigene Vorteil gestand allmählich einen anderweitigen Ersatz des zugefügten Schadens zu. Sobald es dann Güter gab, die dem Menschen von dauerndem Wert waren, brachte dieser Zustand den Tausch derselben mit sich. Der eine gab dem andern ein Gut, welches dem dafür Eingetauschten zu entsprechen schien, insofern es einen gleichartigen Wert für beide hatte.

Solche Güter wurden dann auch als Ersatz gebraucht für nicht gewährte Leistungen oder als Entschädigung für erduldete Unbilden. Auf diese Weise wurde die Rache ermäßigt, zumal seit Gemeinwesen zu entstehen begannen. Eine jede Entschädigung, jede nicht gewährte Leistung erhielt nun ihr Äquivalent. Dieses bestand nicht immer in sachlichen Gütern. Das war z. B. unmöglich, wo das versprochene Gut nicht gewährt werden konnte.

Wodurch entschädigte sich nun der geschädigte Gläubiger für diesen Fall? Durch den Genuß der Vergewaltigung. Dieser hat in der Form der Knechtung und Marterung bei allen Naturvölkern bestanden. Erst im Laufe längerer Zeit wurden die Shylocks seltener.

Nietzsche hat gewiß nicht unrecht, wenn er die Schadenfreude als eine der größten Freuden ursprünglicher Naturvölker ansieht. Er macht unter anderem darauf aufmerksam, daß noch das christliche Mittelalter hohe Festlichkeiten der. Kirche und des Thrones gern durch Autodafés und dergl. gefeiert hat.

Doch genug: die Aussicht auf Vergeltung mußte gerade auf diesem grausamen Wege notwendig dazu führen, dem unkultivierten Menschen ein dauernderes Gedächtnis für sittliche Beziehungen, Verpflichtungen und dergl. zu schaffen, wenn nicht im großen überhaupt erst zu ermöglichen.

Auf diesem geschichtlichen Wege mögen daher Vorstellungen wie Schuld, Pflicht, Schulderlaß und ähnliche zuerst erwacht 
sein, dio sich dann zunehmend verfeinerten. Selbst die Sündenvorgobung Jaluves orscheint im Alten T'estamente nachweisbar zunächst nicht als Erlaß der Schuld im ethischen Sinne, sondern der Strafe.

Auf diese Weise war das anfangs körperliche Empfindungsund Gefühlsinteresse für die Urmenschen ein Hauptanlaß, auf welchen hin sich ein dauernderes Gedächtnis und Gewissen für das Rechtverhalten der Menschen untereinander gebildet und entwickelt hat.

Indem Nietzsche die soeben angedeutete Entstehungsart der sittlichen Vorstellungen insbesondere für die Schuld und das Schuldgefühl aufzeigt, stützt er sich wiederum, auch auf deutschem Gebiete, auf die Etymologie. In der Tat gibt der Gang der Bedeutungsentwicklung der Wörter vielfach Aufschluß über die Bildung der sittlichen Vorstellungen. Von dieser fruchtbaren Seite ist die Sache immer noch zu wenig in Angriff genommen worden. Gleich allen Vorstellungen überhaupt sind die sittlichen, wie auch die Sprachforschung lehrt, in sinnlicher, handgreiflicher, massiver Form entstanden. Dementsprechend gehen die Bedeutungen der Wörter stets von konkreten Beziehungen und Verhältnissen aus und werden erst allmählich auf abstraktere Gebiete übertragen. So muß es sich für den Begriff der Schuld auch sprachlich nachweisen lassen, daß das Wort dafür anfangs ein äußeres Verhältnis bezeichnet hat, und daß sich diese Bedeutung später vergeistigte. "Schulden" im weitesten Sinne waren die er'ste "Schuld“. Auch die deutsche Sprache versteht unter der Schuld zunächst eine äußere Leistung, zu der man verbindlich gemacht ist. Zuerst natürlich an Gütern, später an Geld und anderem.

Auf diesem Wege müssen sich, wenigstens im allgemeinen, die ersten festen "rechtlichen und sittlichen Ordnungen gebildet haben. Dies bezeugen auch viele ursprüngliche Bräuche der Naturvölker. So entstanden allmählich bestimmte Vorstellungen von Recht und Unrecht. Das Gemeinwesen organisierte sich nach und nach, ein jedes in seiner individuellen Art.' In dieser drückte sich, soweit sie spontan entstand, der derzeitige Wille einer bestimmten Gemeinschaft mehr oder weniger vollkommen aus. Aus dem Gewohn. 
heitsrecht wurde, mit der Weiterbildung zum Staate, das Satzungsrecht. Endlich übernahm dieser selbst diejenigen Bildungen des Privatrechts, welche im Interesse des Ganzen lagen. Er regelte die Kaufs-, Vertrags- und Strafordnungen. Er züchtigte den, der gegen die nunmehr entstandenen Staatsgesetze grundsätzlich verstieß, als „Verbrecher“, indem er ihn als Friedlosen aus dem Ge$n u ß$ des Gemeinwesens ausschloß und vogelfrei machte.

Auch das hat Nietzsche in der Hauptsache gewiß richtig dargestellt. Nur darf man auch hier wieder nicht mit ihm Anlaß und Ursache verwechseln und darf die Ursache nicht bloß einseitig egoistisch fassen. Der Anlaß solcher Entwicklung von Sitten, Ordnungen, Gesetzen lag in äußeren Nötigungen des Verkehrs der Menschen untereinander. Die Ursache, sich zù diesem zu verstehen, war zunächst überwiegend der eigene Vorteil. Dies beides aber trug dazu bei, daß die Menschen den tiefsten Grund ihres Wesens entwickelten, in welchem neben dem Egoismus auch der Altruismus schlummerte. Je mebr dieser erwachte, desto deutlicher ging ihnen eine, zunächst instinktive, Ahnung davon auf, daß sie in der Förderung auch der fremden Interessen mittelbar ihre eigenen betrieben. Sie fühlten sich zwar nicht als dieselben mit den anderen, aber doch in ihrem tiefsten Wesen mit ihnen verwandt. Zuletzt gewabrten sie immer mehr, daß sie auch ihr eigenes Ziel nur in der Wahrung höherer Ziele der umfassenderen Gemeinschaft, zu der sie gehörten, erreichen konnten.

Doch kehren wir noch mit einigen Worten zu der Entstehung der ersten Rechtsordnungen zurück. Ursprünglich schrieben auch die Gemeinwesen überall mit Blut und straften nach höchst äußerlichen Maßstäben. Zeuge dafür ist das "Auge um Auge, Zahn um Zahn" der Babylonier und Juden, das sogenannte „jus talionis" der Römer und ähnliches. Erst nach und nach wurden die Gesetze milder. Der Weg, auf welchem sich in der Öffentlichkeit Sitte und Recht entwickelt und befestigt haben, war ein steiler, strenger und grausamer. Noch heute liegt hier und da ein Duft von dem nicht gar lange erloschenen Brande der Scheiterhaufen in der Luft. Auch weiß man, wie unmenschlich vor hundert bis zweihundert Jahren z. B. Taubstumme, Epileptische und andere abnorme Kranke, 
sogar von höher ontwickelten Staatswesen, behandelt worden sind. Die Aufhebung der Lcibeigenschaft datiert, selbst in Deutschland, teii weise erst aus dem Anfange des vorigen Jahrhunderts. Die Beförderung widerspenstiger oder auch nur der Regierung mißliebiger Russen nach Sibirien, d. h. in Jammer und Tod, ist bis heute an der Tagesordnung. Die Duldung von Leuten, die religiös oder politisch eine andere Überzeugung haben, ist zwar im allgemeinen jetzt in den kultivierten Ländern gesetzlich geschützt, aber als sittliche Notwendigkeit keineswegs durchgreifend in das Gewissen mancher gesellschaftlichen Schichten übergegangen. Auch heute pflegt der Weg jeglichen Fortschritts, wenigstens auf dem Gebiete der höchsten menschlichen Interessen, wenn nicht mit dem Blut, doch mit den Tränen der Märtyrer befeuchtet zu werden.

7. Das Familienleben als Quelle sittlicher Vorstellungen.

Obwohl diese bitteren Wahrheiten sich nicht bestreiten lassen, muß doch ausdrücklich hervorgehoben werden, daß Nietzsche hier wiederum mit Unrecht von der bereits erwähnten Stätte ursprünglichster Sittlichkeit absieht. Ich meine das Leben der durch dasselbe Blut verbundenen.F amiliengemeinschaft. Zwar gab es auch hier in frühen Zeiten manche drakonische Maßregel. Ich erinnere nur an das Recht der Väter, über Leben und Tod ihrer Abkömmlinge, an die Kinderaussetzungen der Spartaner, an die Mädchentötung der Chinesen: Dennoch entwickelten sich in der Familie frühzeitig Keime der zartesten, selbstlosesten Liebe, der Ehrfurcht und des Gehorsams bis zu gegenseitiger Aufopferung. Nicht bloß in der Beziehung der Mutter zum Kinde, in dem Verhältnis kindlicher Pietät gegenüber den Erzeugern und Vorfahren, sondern auch in dem gegenseitigen Verkehr der Gatten. Gestaltet sich doch die eheliche Liebe selbst schon bei manchen Tierarten zu einer Gemeinschaft wechselseitiger Treue in dauernder, sogar monogamischer Verbindung.

Die in der Familie angelegten und ausgebildeten altruistischen Eigenschaften betätigten sich dann in immer umfassenderen Glie- 
derungen, wenn auch in abgeschwächter Gestalt, bis sie endlich die kaum mehr sichtbare, aber nicht verschwundene tiefste Gemütsgrundlage für Stamm und Volk wurden.

Das Gefühl für entferntere sittliche Beziehungen freilich weitete sich nur langsam aus. Wie lange Zeit ist doch der Fremde als der natürliche Feind angesehen worden! „Hostis“, Feind und "hospes", Gast, gehen bekanntlich auf denselben Stamm zurück. Man haßte den Fremden, fürchtete ihn auch wohl und suchte ihn jedenfalls unschädlich zu machen. Die Sitte der Fremdenopferung, wie sie uns Goethe aus den Anfängen der griechischen Kultur bei den Tauriern malt, war bei den ursprünglichen Völkern allgemein. Auch pflegten diese anfangs alle, die nicht ihre Sprache redeten, nicht für volle Menschen zu nehmen. Sogar für den echten Griechen der klassischen Zeit behielt das Wort „Barbar" einen Nachklang ursprünglicher Zustände. Barbar heißt "Zwitscherer". Völker anderer Zunge hatten hiernach gar keine eigentliche Sprache, sondern nur den Vögeln ähnliche Naturlaute. Und für den Philosophen Aristoteles, der die Blïte griechischer Kultur hinter sich hatte, war der Sklave ein mit Verstand ausgerüstetes Zugtier.

Aus alledem läßt sich verstehen, daß der Altruismus, welcher in der Familie von jeher heimisch war, erst langsam und spät von nennbarem Einfluß für die Ordnungen gewesen sein kann; die den Verkehr größerer Gemeinschaften untereinander regelten. $\mathrm{Zu}$ der Bildung des eigentlichen Rechts war ein zwingender Anlaß nur vorhanden, wo ebenbürtige Parteien mit einander verkehrten, die durch keine unmittelbaren Bande des Bluts oder der natürlichen Pietät verbunden waren, und in deren Interesse es dennoch lag, sich in ein erträgliches Einvernehmen zu setzen. Dieses Recht mag sich dann vielfach nach Vorbild und Analogie der Familiensitte gebildet haben. Jedenfalls aber war der Leitfaden für seine Entwicklung nicht so sehr der Altruismus, wie der Egoismus.

8. Abschließender Rückblick auf die Entstehung der sittlichen Vorstellungen.

Blicken wir von hier aus auf die Stellang zurück, welche Kant dem Sittengesetze gibt. Er begründet die Geltung desselben 
fïr den Menschen wesentlich damit, daß es der Natur desselben nicht entspricht. Darauf wärde man so ohne weiteres ebensogut dic Nichtgeltung begründen können. Wie viel näher liegt heutzutage die Erklärung seiner Autorität, der Gewissensurteile u. s. w. aus ererbter Gefühlsgewohnheit! Diese Art zu fühlen hatte sich im Laufe der Generationen verfestigt und "einverseelt" und geht schließlich auf $\mathrm{W}$ erturteile früherer Generationen zurück. Wie stark dieser Einfluß sein mußte, darauf kann man noch heute an dem Druck der öffentlichen Meinung der derzeitigen Umgebung die Probe machen.

Aber auch diese Erklärung ist einseitig. Denn sie läßt die dem Menschen innerlich einwohnende und angeborene sittliche Anlage unbeachtet oder doch unerklärt. Ja wenn man tiefer greift, vernachlässigt sie damit den Zusammenhang des Sittengesetzes mit der Gottheit, welche offenbar Kant vor allem am Herzen lag. Und hier tritt in der Tat die Wahrheit seines Strebens hervor und der Grund, weshalb noch heuzutage nicht wenige fromme Denker an der Ansicht des Königsberger Philosophen festhalten.

Will man nun aber das Göttliche mit dem zweifellos geschichtlich entwickelten Sittengesetz und Gewissen in Beziehung setzen, dann muß man noch tiefer graben. Man muß auf die Harmonie des subjektiven und objektiven Lebens zurückgehen, worauf die inner- und außerseelische Wirksamkeit der Gottheit eben mittels der Entwicklung der Menschen und der Menschheit hinzielt. Nur so läßt sich Gesetz, Wesen und Bestimmung des Menschen mit dem Gesetz von Welt und Menschheit ausgleichen.

Nach alledem wird man vielleicht hauptsächlich vier ursprüngliche Entstehungsorte sittlicher Gefühle und Vorstellungen (in weiterem Sinne) annehmen dürfen. Sie entstanden erstlich in der Familie, sodann auf der Seite der Herrschenden, wiederum von seiten der geknechteten oder untergeordneten Einzelnen, wie auch Stämme und Völker, endlich als spontaner Ausdruck des "Gemeinwillens", d. h. des Willens der Gemeinwesen in mehr oder weniger verfaßten Stämmen oder Völkern.

Wenn wir noch einmal die Hauptpunkte der Entwicklung der sittlichen Vorstellungen überblicken, so ergibt sich also kurz fol- 
gender Gang. Sobald der wechselseitige Egoismus der Einzelnen und Stämme klug wurde, und soweit er Vernunft annahm, war er genötigt, dem individuellen Belieben im engeren und weiteren Sinne gegenseitige Schranken zu ziehen und Bedingungen einzugehen, unter denen überhaupt der fernere Bestand und ein unschädlicher, selbst förderlicher Verkehr ermöglicht und gesichert wurde. Daraus erwuchsen mithin soziale Verbindungen über die Familie hinaus, die, wenigstens im allgemeinen, zunahmen und sich immer mehr konsolidierten. Diese erweiterten Gemeinschaften schränkten dann die individuelle Selbstliebe zunehmend zu Gunsten der Gesellschaft ein.

Soweit der objektive Gang der Entstehung von Recht und Sittlichkeit ganz allgemein. Erst innerhalb dieser Gemeinschaftsformen und auf Anlaß derselben bildeten sich die Einzelseelen zu einer höheren, innerlichen, subjektiven Sittlichlieit empor. Freilich war diese von Anfang an angelegt und zeitigte ihre ersten Früchte schon früh im Familienleben. Der Genuß derselben aber kam weiteren Kreisen zunächst nicht zugute. Erst in der Gesellschaft wird das für diese bestimmte menschliche Wesen wahrhaft menschlich. Erst die heftige Reibung des Individuums mit ihr unterwirft den natürlichen Egoismus immer mehr und immer umfassender dem Altruismus. Sie ist es, die aus dem harten Steire der Selbstsucht stets hellere Funken selbstloser Gesinnung herausschlägt. Die soziale Hemmung der Selbstliebe pflegt in der Geschichte die Menschenliebe "auszulösen“. So wird der Streit zum Vater der Liebe. Die Mutter aber ist die mitgegebene altruistische Anlage. Alle menschlichen Anlagen jedoch schlummern zunächst. So auch die sittliche. Ehe sie durch jenen Anlaß befruchtet ist, bringt sie ihr eingeborenes herrliches Erzeugnis nicht ans Licht.

Die allmähliche Aufnahme des Gemeingeistes in den Individualgeist vermenschliclst und versittlicht auf diesem Wege den Einzelnen, erhebt ihn über die Tierheit und erweckt in ihm zunehmend den Sinn echter Humanität.

In diesem Gemeingeiste aber und mittels desselben erzieht der Weltgeist selber die Völker und Menschen mehr und mehr zu 
altruistischem Verhalten gegen alles, was Mensch heißt, zuletzt gegen allos Lebendige. So wird der einzelne Mensch stets tiefer von den höchsten Funktionen des Allgeistes durchlebt. Dieser schließt die Einzelnen zusammen und bildet ihr Fürsich in ein Füreinander um. Dor Geist der Liebe führt den zunehmend vergeistigten Menschen seiner göttlichen Bestimmung, in der Hingabe an das Ganze, an den, der es durchdringt und durchwaltet, entgegen.

Dies ist hier nicht weiter auszuführen. Mir lag nur daran, darauf hinzuweisen, daß jede zukünftige Ethik mit Nietzsches Anschaungen zu rechnen hat. Nur müssen wir uns dabei eben vor seinen Einseitigkeiten hüten. Was sich ihm zuerst aufhellte, und worin seine schöpferische Eigenart lag, in dessen Lichte erschien ihm vielfach das Ganze.

Der Individualismus $m u B$ in seiner Bedeutung auch für das sittliche Gebiet anerkannt werden. Um so nötiger aber ist es dann, die tatsächlich vorhandene und zunehmend entwickelte altruistische Anlage nicht zu verkennen, sondern daneben zu würdigen. Denn sie macht den Menschen erst wahrhaft zum Menschen.

Wir müssen in dieser Entwicklung des Menschen und der Menschheit aus vorwiegendem Egoismus zu vorwiegendem Altruismus, ja Universalismus, wenigstens im großen, einen allmählichen Fortschritt zum Ideale anerkennen, trotz mancher Rückgänge und Rückfälle in Einzelnen, in Völkern und Zeiten. Die Erkenntnis des Sittlichen ist in zunehmender Verfeinerung begriffen, zumal, je mehr die Menschheit theoretisch und praktisch in eine gemeinsame „Weltgeschichte" eintritt.

Die höchste Kraft zu völliger Versittlichung der Einzelnen und der Völker -kann jedoch durch die richtige. Erkenntnis allein nicht gewonnen werden. Sie quillt, wenn überhaupt, einzig aus dem tiefsten Gemütsleben: aus dem Zusammenhang des Weltgeistes mit dem innersten Lebensgrunde. So ist es die religiöse Beziehung des Menschen, die allein die Vergöttlichung menschlicher Gesinnung zu bewirken vermag.

Voraussetzung dafür ist die Entwicklung der Menschheitsgeschichte bis auf einen bestimmten Höhepunkt. Denn hieran ist 
die Ausreifung wabren Menschentums gebunden. Erst dann ist die Zeit erfüllt, um nunmehr den Himmelsweg höchster sittlicher Ideale betreten zu können. Die Erfüllung der Zeit aber ist mit demjenigen eingetreten, der, in seinem Herzen von wahrhaft göttlicher Gesinnung durchdrungen, der Menschheit das Heil in vollendeter Gottes- und Menschenliebe persönlich dargelebt hat. Dadurch hat er ihr nicht nur das Ziel unendlicher Vergöttlichung aufgestellt, sondern sie in Kraft seines Geistes befähigt, ihm mehr und mehr zuzuschreiten.

(Anm. Nietzsche hat seinen einseitigen Individualismus mit der ihm eigenen radikalen Konsequenz nicht nur auf dem sittlichen, sondern auch auf dem intellektuellen und ästhetischen Gebiete geltend gemacht. Davon durfte ich indessen hier absehen, wo es mir nur um die sittlichen Vorstellungen zu tun war.) 
